\section{Aspirin as a painkiller in patients with a high cardiovascular risk profile}

Rainer Spiegel

In their Review article (Cardiovascular effects and safety of (non-aspirin) NSAIDs. Nat. Rev. Cardiol. https://doi.org/10.1038/s41569-0200366-z (2020)) $)^{1}$, Schjerning and colleagues focus on the cardiovascular safety of nonsteroidal anti-inflammatory drugs (NSAIDs) for pain management. I entirely agree with their conclusion in favour of multimodal pain management in patients with a high cardiovascular risk profile, including the need for physiotherapy, weight management and exercise. Schjerning and colleagues thoughtfully point out the pharmacodynamic interactions between aspirin and both naproxen and ibuprofen ${ }^{1}$. In addition, the authors outline the risk of myocardial infarction in relation to the administration of nonselective NSAIDs, such as diclofenac, ibuprofen and naproxen ${ }^{1-3}$, or selective cyclooxygenase 2 (COX2) inhibitors ${ }^{1,4}$. I have seen many patients with a high cardiovascular risk profile who were taking nonselective NSAIDs to control their pain and entirely agree with the authors' warnings. With the current opioid crisis in the USA, the number of these patients might even rise because NSAIDs have been shown to reduce the opioid doses required for pain management ${ }^{5}$.

In the authors' conclusion, however, I note that analgesic-dose aspirin is not mentioned as an alternative treatment in patients with a high cardiovascular risk profile, particularly in patients who are already taking aspirin to prevent myocardial infarction. This approach would not only avoid pharmacodynamic interactions between aspirin and other NSAIDs but would also circumvent the risk of myocardial infarction associated with the use of diclofenac, ibuprofen, naproxen and selective COX2 inhibitors ${ }^{2-4}$.

In summary, rather than adding any of the aforementioned non-selective NSAIDs or selective COX2 inhibitors to the administration of prophylactic aspirin in patients with a high cardiovascular risk profile, would it not make sense to opt for aspirin monotherapy and to increase its dose from a prophylactic to an analgesic dose, if the aim is to control pain? Depending on the nature of the pain, analgesic-dose aspirin might be more potent than alternatives such as paracetamol (acetaminophen). One would obviously have to weigh the risks and the benefits and carefully consider the indication; for example, the antiplatelet effect of aspirin might substantially increase the risk of bleeding during a subsequent operation, and aspirin use carries a risk of allergic reactions. In addition, the feasibility and safety of analgesic-dose aspirin for pain management in patients with a high cardiovascular risk profile would have to be shown in randomized, prospective trials, which have not currently been performed.

There is a reply to this letter by Schjerning, A.-M. et al. Nat. Rev. Cardiol. https://doi.org/ 10.1038/s41569-020-0399-3 (2020).
We thank Rainer Spiegel for his Correspondence (Aspirin as a painkiller in patients with a high cardiovascular risk profile. Nat. Rev. Cardiol. https://doi.org/10.1038/s41569020-0398-4 (2020)) $)^{1}$ on our Review (Cardiovascular effects and safety of (non-aspirin) NSAIDs. Nat. Rev. Cardiol. https://doi.org/ 10.1038/s41569-020-0366-z (2020)) 2. We share his concerns about the lack of effective and safe analgesic drugs and the potential increase in the use of nonsteroidal antiinflammatory drugs (NSAIDs) in the wake of the opioid crisis in the USA.

Spiegel suggests that analgesic-dose aspirin might have a cardiovascular benefit as an alternative analgesic treatment to NSAIDs. In our Review, the primary focus was on commonly prescribed NSAIDs, and analgesic-dose aspirin is not commonly prescribed in the countries in which we practise, Denmark (Danish Health Data Authority) and the $\mathrm{UK}^{3}$, although aspirin is widely available without prescription. We are unaware of good evidence demonstrating a better cardiovascular benefit-risk balance with analgesic-dose aspirin than with NSAIDs. Industry-sponsored, randomized clinical trials and independent epidemiological investigations have focused on the gastrointestinal effects of analgesic-dose aspirin ${ }^{4-6}$.
Rainer Spiegel iD

BG Trauma Center, Division of Anesthesiology. Intensive Care and Pain Management, University of Tübingen, Tübingen, Germany. e-mail: rainer_spiegel@hotmail.com https://doi.org/10.1038/s41569-020-0398-4

Schjerning, A.-M. et al. Cardiovascular effects and safety of (non-aspirin) NSAIDs. Nat. Rev. Cardiol. https://doi.org/10.1038/s41569-020-0366-z (2020).

2. Bally, M. et al. Risk of acute myocardial infarction with NSAIDs in real world use: Bayesian meta-analysis of individual patient data. BMJ 357, j1909 (2017).

Masclee, G. M. C. et al. Risk of acute myocardial infarction during use of individual NSAIDs: a nested case-control study from the SOS project. PLoS One 13, e0204746 (2018).

4. Coxib and traditional NSAID Trialists' (CNT) Collaboration. Vascular and upper gastrointestinal effects of non- steroidal anti-inflammatory drugs: meta-analyses of individual participant data from randomised trials. Lancet 382, 769-779 (2013).

Martinez, L. et al. Perioperative opioid-sparing strategies: utility of conventional NSAIDs in adults. Clin. Ther. 41, 2612-2628 (2019)

\section{Competing interests}

The author owns shares in the respirator company Dräger but declares no competing interests in relation to this Correspondence.

\title{
Reply to 'Aspirin as a painkiller in patients with a high cardiovascular risk profile'
}

\section{Anne-Marie Schjerning, Patricia McGettigan (D) and Gunnar H. Gislason (D)}

From a practical-use perspective, the duration of analgesia with aspirin is short, and dosing every $3-4 \mathrm{~h}$ to a maximum dose of $3.6 \mathrm{~g}$ per $24 \mathrm{~h}$ is recommended ${ }^{7}$. Therefore, if aspirin were to be used for analgesia, problems with adherence and breakthrough pain are likely ${ }^{7}$. Importantly, an overdose of aspirin is highly toxic. A community-based, randomized, industry-sponsored study reported that aspirin was unlikely to be superior to ibuprofen as an analgesic and was associated with more adverse effects overall ${ }^{8}$; although, in another industry-sponsored study, analgesic-dose aspirin was reported to be well tolerated during short-term use (median 3 days) ${ }^{4}$.

In summary, we currently find no strong evidence in terms of the cardiovascular benefit-risk balance to support a recommendation for the use of analgesic-dose aspirin as an alternative to other NSAIDs. We agree with Spiegel that the feasibility and safety of analgesic-dose aspirin for pain management in patients with a high cardiovascular risk profile need to be tested in prospective, randomized trials.

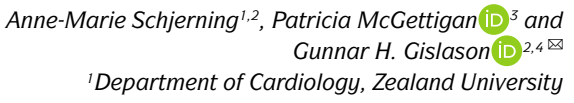
Gunnar H. Gislason (iD 2,4凶 ${ }^{1}$ Department of Cardiology, Zealand University Hospital, Roskilde, Denmark. 


\section{CORRESPONDENCE}

${ }^{2}$ The Danish Heart Foundation, Copenhagen, Denmark. ${ }^{3}$ William Harvey Research Institute, Barts and the London School of Medicine and Dentistry, Queen Mary University of London, London, UK. ${ }^{4}$ Department of Cardiology, Copenhagen University Hospital Herlev and Gentofte, Hellerup, Denmark. 凶e-mail: gg@heart.dk

https://doi.org/10.1038/s41569-020-0399-3

1. Spiegel, R. Aspirin as a painkiller in patients with a high cardiovascular risk profile. Nat. Rev. Cardiol. https://doi.org/10.1038/s41569-020-0398-4 (2020).

2. Schjerning, A. M., McGettigan, P. \& Gislason, G. Cardiovascular effects and safety of (non-aspirin)
NSAIDs. Nat. Rev. Cardiol. https://doi.org/10.1038/ s41569-020-0366-z (2020).

3. NHS Digital. Prescription cost analysis - England 2018 [PAS]. NHS Digital https://digital.nhs.uk/ data-and-information/publications/statistical/ prescription-cost-analysis/2018 (2019).

4. Forder, S., Voelker, M. \& Lanas, A. Gastrointestinal safety of aspirin for a high-dose, multiple-day treatment regimen: a meta-analysis of three randomized controlled trials. Drugs R D 16, 263-269 (2016)

5. Huang, E. S., Strate, L. L., Ho, W. W., Lee, S. S. \& Chan, A. T. Long-term use of aspirin and the risk of gastrointestinal bleeding. Am. J. Med. 124, 426-433 (2011)

6. Henry, D. et al. Variability in risk of gastrointestinal complications with individual non-steroidal anti-inflammatory drugs: results of a collaborative meta-analysis. BMJ 312, 1563-1566 (1996).

7. Electronic Medicines Compendium. Aspirin tablets BP $300 \mathrm{mg}$ (POM). Electronic Medicines Compendium https://www.medicines.org.uk/emc/product/5712/ smpc\# (2020).

8. Moore, N. et al. The PAIN study: Paracetamol, Aspirin and Ibuprofen New tolerability study. Clin. Drug Investig. 18, 89-98 (1999).

\section{Competing interests}

The authors declare no competing interests.

RELATED LINK

Danish Health Data Authority: https://medstat.dk/en 